\title{
Métodos de classificação aplicados à detecção automática de Estilos de Aprendizagem em um ambiente real de ensino
}

\author{
Lucas Daniel Ferreira ${ }^{1}$, Murilo G. Gazzola ${ }^{1}$, Deborah V. Ferrari ${ }^{2}$, \\ Marina M. Zupelari' ${ }^{2}$, Paula Paiva ${ }^{2}$, Jose F Rodrigues-Jr ${ }^{1}$ \\ ${ }^{1}$ Instituto de Ciências Matemáticas e de Computação - Universidade de São Paulo (USP) \\ Caixa Postal 13566-590 - São Carlos - SP - Brasil \\ ${ }^{2}$ Faculdade de Odontologia de Bauru - Universidade de São Paulo (USP) \\ Caixa Postal 17012-901 - Bauru - SP - Brazil \\ \{lucas.danfer, deborahferrari,mmorettin, paulapaiva\}@usp.br \\ \{mgazzola, junio\}@icmc.usp.br
}

\begin{abstract}
The automatic detection of learning styles is an active research topic in the field of technology applied to education, however, most of existing works are not conclusive in relation to the different classification methods applied in this scenario. In this work, therefore, we evaluate four machine learning algorithms, specifically for the classification task, in order to identify the Learning Styles of the students in a real electronic learning environment, according to the Felder-Silverman model. As a result, the experiments indicated that a single method is not sufficient for the correct classification in all the dimensions, and that the best scenario contemplates the use of at least two different classifiers.

Resumo. A detecção automática de Estilos de Aprendizagem é um ativo tópico de pesquisa na área de computação aplicada ao contexto educacional, porém, grande parte dos trabalhos existentes não é conclusiva com relação a diferentes métodos de classificação aplicados neste cenário. Neste trabalho, portanto, são avaliados quatro algoritmos de aprendizado de máquina, especificamente para a tarefa de classificação, a fim de se identificar os Estilos de Aprendizagem dos estudantes em um ambiente real de aprendizado eletrônico, de acordo com o modelo de Felder e Silverman. Como resultado, os experimentos indicaram que um único método não é suficiente para a correta classificação em todas as dimensões, sendo que o melhor cenário contempla o uso de, pelo menos, dois classificadores diferentes.
\end{abstract}

\section{Introdução}

Cada estudante, individualmente, possui habilidades, necessidades, conhecimentos prévios e motivações próprias, e estas características são fundamentais para determinar se um indivíduo encontra mais dificuldades do que outros para aprender em certo modelo de ensino [Graf 2007]. De fato, conforme explorado no trabalho de [Coffield et al. 2004], diversos estudos nas áreas de psicologia cognitiva e pedagogia indicam que cada pessoa possui diferentes maneiras de captar, processar e organizar informações durante o aprendizado, o que fundamenta o conceito de Estilos de Aprendizagem.

O conceito de Estilos de Aprendizagem motivou diversas pesquisas na área da computação aplicada à educação, pois a construção de um modelo do perfil dos alunos 
pode melhorar os processos de ensino e aprendizagem de diversas maneiras. Um exemplo de aplicação é a recomendação de conteúdo personalizado para cada estudante de acordo com suas preferências. Assume-se que esta abordagem de ensino pode tornar o ensino mais proveitoso e melhorar o desempenho dos alunos.

É premissa deste trabalho, e de outros existentes ([García et al. 2007], [Graf et al. 2009], [Dorça et al. 2011], [Dung and Florea 2012] e [Sena 2016]) que uma abordagem de detecção automática dos estilos de aprendizagem pode proporcionar melhores resultados no que se refere ao aproveitamento intelectual dos alunos. Assim, o objetivo deste trabalho é explorar diferentes técnicas de aprendizado de máquina supervisionado, relacionadas especificamente à tarefa de classificação aplicadas à detecção automática de EAs em um ambiente virtual de ensino. Para isso, serão experimentados algoritmos de diferentes paradigmas na tarefa de classificação de estudantes com base nas interações realizadas durante o uso de um sistema de gestão do aprendizado. Ao fim do estudo, espera-se encontrar uma combinação de técnicas e configurações de dados que atinja resultados satisfatórios quando comparada aos trabalhos correlatos no que diz respeito à efetividade da classificação.

No desenvolvimento do trabalho, foi usado como estudo de caso um curso de especialização na área de Fonoaudiologia (Reabilitação Auditiva), onde os recursos educacionais são oferecidos integralmente pelo sistema Moodle. Dentre os diversos modelos propostos na literatura, optou-se por usar o Modelo de Estilos de Aprendizagem de Felder-Silverman [Felder et al. 1988], o qual sintetiza descobertas de diversos estudos em quatro dimensões específicas: visual/verbal, ativo/reflexivo, sensorial/intuitivo, sequencial/global. Este artigo está organizado da seguinte maneira: Na Seção 2 são apresentados os principais conceitos necessários para a compreensão do trabalho. Na Seção 3 são apresentados os trabalhos com propostas semelhantes à deste estudo. A Seção 4 descreve a metodologia implementada e por fim, na Seção 5, é descrita a avaliação experimental. Considerações finais, conclusões e direcionamentos futuros são discutidos na Seção 6.

\section{FUNDAMENTAÇÃO TEÓRICA}

\subsection{Estilos de Aprendizagem}

O termo "Estilos de Aprendizagem" não possui uma definição específica. A definição mais comumente aceita é a de Keefe [Keefe 1979], que considera os Estilos de Aprendizagem como "uma composição de características cognitivas, afetivas e fatores fisiológicos que servem como indicadores relativamente estáveis de como um aluno percebe, interage e responde ao ambiente de aprendizagem". De maneira geral, os Estilos de Aprendizagem referem-se aos métodos que um estudante aplica com frequência para observar o ambiente, captar informações, as processar, compreender seu significado e então transformá-las em conhecimento. Embora trabalhos recentes apresentem críticas à teoria dos Estilos de Aprendizagem [Kirschner 2017], neste trabalho acredita-se que esta seja uma ferramenta efetiva para classificar aprendizes online, visto que em cada modelo de EA são propostos comportamentos rastreáveis relacionados a cada perfil de estudante. Inúmeros autores propuseram modelos para classificar Estilos de Aprendizagem capazes de mapear o comportamento de estudantes. Neste trabalho, é explorado o Modelo de Estilos de Aprendizagem de Felder e Silverman (FSLSM) [Felder et al. 1988], que será detalhado a seguir. Uma vasta revisão acerca de modelos de estilos de aprendizagem está disponível no trabalho de Coffield et al. [Coffield et al. 2004]. 
VI Congresso Brasileiro de Informática na Educação (CBIE 2017)

Anais do XXVIII Simpósio Brasileiro de Informática na Educação (SBIE 2017)

\subsection{Modelo de Felder-Silverman (FSLSM)}

O modelo de Felder e Silverman [Felder et al. 1988] se refere a uma compilação de modelos propostos na literatura a fim de se alcançar um modelo de maior amplitude e possibilidades. Felder e Silverman sintetizaram descobertas de diversos estudos que resultaram em um modelo de estilos de aprendizagem composto por quatro dimensões distintas, que são detalhadas a seguir.

- Processamento (ativos x reflexivos): os estudantes ativos precisam experimentar para compreender, poderão iniciar as tarefas prematuramente e gostam de participar de trabalhos em grupo. Já os estudantes reflexivos precisam compreender para experimentar, demoram a iniciar as atividades e preferem trabalhos individuais.

- Captação (visuais x verbais): indivíduos visuais possuem melhor captação de informações que se apresentam de forma gráfica, tais como ilustrações, figuras, diagramas, fluxogramas, esquemas e mapas. Em contrapartida, os verbais estão relacionados às propriedades de leitura e audição.

- Organização (sequenciais $\mathbf{x}$ globais): estudantes sequenciais organizam a informação a partir do detalhamento para compreender informações mais generalizadas. Por outro lado, estudantes globais organizam a informação a partir de regras gerais, para que a partir destas cheguem ao nível de detalhe.

- Percepção (sensoriais $\mathbf{x}$ intuitivos): indivíduos intuitivos possuem grande capacidade para distinguir e interpretar símbolos e textos quando comparados aos sensoriais. Por outro lado, os indivíduos sensoriais tomam decisões e percebem as informações baseando-se fortemente nos sentidos (visão, audição, tato, olfato).

O modelo de Felder-Silverman foi escolhido por diversas razões. Uma delas é o fato de que os autores agruparam em um único modelo importantes descobertas de outros autores já consolidados neste campo de investigação. Outra característica importante é a praticidade para se identificar o estilo de um estudante, visto que Felder e Soloman desenvolveram um questionário contendo 44 questões (11 questões para cada dimensão), denominado Index of Learning Styles (ILS) [Soloman and Felder 2005], que aponta as preferências de cada indivíduo de acordo com o modelo proposto. Por fim, a escolha do modelo de Felder-Silverman justifica-se por este ser um dos modelos mais adotados em trabalhos de modelagem automática de estudantes [Feldman et al. 2015] [Truong 2016], além de já ser incorporado a diversos sistemas adaptativos, demonstrando bons resultados quanto à classificação de perfis e recomendação de conteúdo.

\section{Trabalhos Relacionados}

Diversas abordagens foram propostas para identificação automática de Estilos de Aprendizagem em ambientes virtuais de ensino. O trabalho de Garcia et al. [García et al. 2007], por exemplo, foi um dos precursores no tema e representou a base para vários outros trabalhos que relacionam redes bayesianas e o modelo de estilos de Felder-Silverman (FSLSM). Neste trabalho os autores aplicaram o Teorema de Bayes e observaram 11 padrões de comportamento em 10 estudantes matriculados em um curso de Inteligência Artificial, oferecido via Web.

Dorça et al. apresentam inúmeras contribuições para o contexto da modelagem automática e dinâmica de estudantes por meio de EA. Em um de seus trabalhos [Dorça et al. 2011], por exemplo, é descrita uma proposta para detectar os Estilos de 
Aprendizagem de estudantes centrada principalmente nos aspectos não determinísticos e não estacionários dos Estilos de Aprendizagem. O foco do trabalho é a investigação de como construir e atualizar gradualmente o modelo dos estudantes, visto que as preferências de cada indivíduo podem mudar de maneira inesperada com o decorrer do tempo. Para a avaliação da proposta, o trabalho de Dorça et al. considera o modelo FSLSM, realizando uma simulação computacional do comportamento de estudantes em um sistema educacional.

O trabalho de Dung e Florea [Dung and Florea 2012] apresenta outra proposta para modelagem automática de estudantes usando o modelo FSLSM, bastante influenciada no trabalho de Graf e Liu [Graf et al. 2009]. Por meio de um experimento com um curso de nove meses sobre Inteligência Artificial, constituído por 204 objetos de aprendizagem, foram avaliados 44 estudantes de graduação que preencheram o questionário ILS como base para avaliação dos métodos de classificação. Com uma abordagem baseada em regras de mapeamento, são considerados o número de visitas e o tempo gasto pelos estudantes em cada objeto de aprendizagem, os quais possuem metadados descritivos.

Dentre os trabalhos contemporâneos, Sena [Sena 2016] propõe uma abordagem probabilística que combina o modelo FSLSM com as técnicas de inferência probabilística dos Modelos Ocultos de Markov (HMM) a fim de realizar o processo de inferência da preferência do aluno por um determinado estilo de aprendizagem. Para isso, matrizes de emissão e transição de estados do modelo de Markov foram criadas por meio do algoritmo de Viterbi. Para avaliar a proposta, os autores realizam uma simulação do comportamento de estudantes em sistemas educacionais, e alcançam bons resultados quanto à detecção de seus EA.

Conforme apontado por Truong [Truong 2016], apesar da variedade de trabalhos conduzidos na área, ainda existem questões em aberto e diversas lacunas a serem investigadas. Uma das questões discutidas é que embora muitos algoritmos de classificação tenham sido explorados - sobretudo Redes Bayesianas e os algoritmos baseados em regras, apenas uma pequena proporção de trabalhos conduz uma análise comparativa entre diferentes métodos de classificação. Portanto, o presente trabalho diferencia-se dos demais apresentados neste capítulo por atender à necessidade de se aplicar diferentes métodos de classificação ao mesmo problema, com o intuito de comparar desempenhos e levantar discussões a respeito dos resultados.

\section{Metodologia}

\subsection{Contexto, Participantes e Objetos de Aprendizagem}

Para a realização deste trabalho, foi avaliado um curso de pós graduação em Reabilitação Auditiva, na área de Fonoaudiologia, oferecido pela Faculdade de Odontologia de Bauru em parceria com o Hospital Samaritano de São Paulo. O curso em questão possui duração de 18 meses, é ministrado integralmente com auxílio do sistema Moodle, e conta com 105 alunos matriculados, oriundos de diversas localidades do Brasil. Dada a separação geográfica, estes estudantes interagem entre si diariamente por meio dos fóruns de discussões e ferramentas de comunicação disponíveis no ambiente virtual. Como parte dos experimentos, os alunos foram solicitados a responder ao questionário ILS no início do curso com o intuito de registrar suas respectivas preferências nas dimensões de processamento, captação, organização, e percepção do modelo de estilos de aprendizagem de Felder-Silverman (FSLSM). Os resultados do questionário são apresentados na Tabela 1, que indica o percentual de preferência dos estudantes para cada dimensão. 
VI Congresso Brasileiro de Informática na Educação (CBIE 2017)

Anais do XXVIII Simpósio Brasileiro de Informática na Educação (SBIE 2017)

Tabela 1. Resultados do questionário ILS

\begin{tabular}{|c|c|c|c|c|c|c|c|}
\hline \multicolumn{2}{|c|}{ Processamento } & \multicolumn{2}{|c|}{ Captação } & \multicolumn{2}{|c|}{ Organização } & \multicolumn{2}{|c|}{ Percepção } \\
\hline Ativo & Reflexivo & Visual & Verbal & Sequencial & Global & Sensorial & Intuitivo \\
\hline $79,38 \%$ & $20,62 \%$ & $53,61 \%$ & $46,39 \%$ & $69,07 \%$ & $30,93 \%$ & $87,63 \%$ & $12,37 \%$ \\
\hline
\end{tabular}

Os registros de uso do Moodle foram coletados entre setembro de 2015 e agosto de 2016. Ao todo, foram avaliadas as interações dos estudantes com 252 recursos educacionais disponibilizados neste período, o que corresponde ao material didático referente aos 16 primeiros capítulos do curso. Estes recursos de aprendizagem são constituídos por materiais de diversas características.

\subsection{Padrões de Comportamento Avaliados}

O modelo de estilos de aprendizagem de Felder e Silverman, assim como os demais modelos propostos na literatura, foi construído com base em conceitos de ensino tradicionais. Portanto, para que seja possível detectar esses Estilos de Aprendizagem de maneira automática e dinâmica em um sistema educativo, é necessário um mapeamento do comportamento dos estudantes em ambientes tradicionais para as ações realizadas no ambiente virtual. Para isso, foi explorado um conjunto de interações capazes de descrever as preferências de cada estudante durante o uso de um sistema educacional. Estes padrões de comportamento foram bastante influenciados pelos trabalhos de Graf [Graf et al. 2009], Garcia et al. [García et al. 2007], e Dung e Florea [Dung and Florea 2012].

Tabela 2. Padrões de Comportamento Avaliados

\begin{tabular}{|c|c|c|}
\hline Categoria & Recursos & Dados Coletados \\
\hline \multirow{4}{*}{ Materiais Didáticos } & Conteúdo Introdutório & Visitas e tempo gasto. \\
\hline & Livro & Visitas, tempo gasto e navegação; \\
\hline & Conteúdo Textual & Visitas e tempo gasto \\
\hline & Conteúdo em Vídeo & Visitas e tempo gasto. \\
\hline \multirow{2}{*}{ Atividades } & Tarefas & Visitas e tempo gasto. \\
\hline & Exercícios & Visitas e tempo gasto. \\
\hline \multirow{3}{*}{ Materiais de Apoio } & Perguntas Frequentes & Visitas e tempo gasto. \\
\hline & Ref. Bibliográficas & Visitas e tempo gasto. \\
\hline & Links Externos & Visitas e tempo gasto. \\
\hline \multirow[t]{2}{*}{ Interação } & Fórum de Discussões & $\begin{array}{l}\text { Visitas ao fórum, visitas aos tópicos, } \\
\text { criação de tópicos, pesquisas, postagens, } \\
\text { média de palavras e tempo gasto. }\end{array}$ \\
\hline & Glossário & Visitas, postagens e tempo gasto. \\
\hline \multirow{2}{*}{ Avaliação } & Auto-avaliação & Visitas, tempo gasto e questões respondidas. \\
\hline & Avaliação do Capítulo & Visitas, tempo gasto e questões respondidas. \\
\hline
\end{tabular}


VI Congresso Brasileiro de Informática na Educação (CBIE 2017)

Anais do XXVIII Simpósio Brasileiro de Informática na Educação (SBIE 2017)

A partir das premissas citadas, foram selecionados os seguintes recursos de interação a serem incorporados a esta experimentação: objetos de aprendizagem referentes à apresentação do material didático, conteúdo introdutório com visões gerais de cada disciplina, materiais de exemplo, vídeos ilustrativos, formulários de auto-avaliação e avaliação de cada capítulo, exercícios, páginas de perguntas frequentes e referências bibliográficas, além dos recursos de comunicação (fórum de discussões e glossário). $\mathrm{Na}$ Tabela 2, são apresentados os padrões de comportamento investigados nesta proposta juntamente com as métricas coletadas pelo sistema.

\subsection{Classificando Estilos de Aprendizagem}

Como já descrito, a tarefa de classificação supervisionada consiste na atribuição de rótulos a determinados objetos não rotulados a partir da observação de suas similaridades com outros objetos já classificados. Neste caso, o primeiro passo para a classificação é modelar o conjunto de dados do domínio ao qual pretende-se aplicar os algoritmos, preparando os exemplos para o processamento. Desta maneira, o conjunto de entrada é composto por uma coleção de exemplos, sendo que cada exemplo é constituído por uma lista de atributos independentes, bem como um atributo classe, que corresponde ao rótulo do exemplo. A partir do processamento dos dados, é construído um conjunto de treinamento que deve servir de entrada para o algoritmo de aprendizado supervisionado. O objetivo do processo de treinamento é obter um modelo de classificador que mapeie as características preditoras de um dado à sua característica de interesse. Em outras palavras, deseja-se encontrar um modelo que relacione os atributos de um dado exemplo com seu atributo classe. Por fim, é realizada a fase de teste do classificador, que busca avaliar a eficácia do modelo gerado, a partir da classificação de novos exemplos ainda não rotulados (conjunto de teste).

\subsection{Software Weka e Validação cruzada}

Em um cenário de aplicação de classificadores para fins científicos, uma alternativa é o uso de ferramentas de apoio de domínio público. Para fins de experimentação, neste trabalho foi usado o pacote de software Weka (Waikato Environment for Knowledge Analysis), o qual implementa uma biblioteca de algoritmos de aprendizado na linguagem Java, e fornece diversas funcionalidades para a extração de medidas, seleção de atributos, e análise de resultados.

Para a avaliação da acurácia de um modelo de classificação foi usada a validação cruzada, a qual pode ser entendida como uma técnica para mensurar a capacidade de generalização de um modelo a partir de um conjunto de dados. O método de validação cruzada denominado $k$-fold consiste em dividir o conjunto total de dados em $k$ subconjuntos do mesmo tamanho. A partir disto, um subconjunto é usado para teste e os $k-1$ restantes são usados para estimação dos parâmetros; calcula-se, então, a acurácia do modelo. Este processo é realizado $k$ vezes alternando-se de forma circular o subconjunto de teste. Ao final das $k$ iterações calcula-se a acurácia sobre os erros encontrados, obtendose assim uma medida confiável sobre a capacidade do modelo de representar o processo gerador dos dados.

\subsection{Protocolo de Experimentação}

Consideradas as etapas do processo de classificação descritas, uma das tarefas fundamentais deste trabalho foi a construção do conjunto de dados para a execução dos experimentos. Os estudantes matriculados no curso correspondem às instâncias do conjunto de 
VI Congresso Brasileiro de Informática na Educação (CBIE 2017)

Anais do XXVIII Simpósio Brasileiro de Informática na Educação (SBIE 2017)

dados, portanto, para cada aluno da amostra, é necessário um vetor de atributos que descreva suas características e um rótulo, fundamental para as fases de treinamento e teste das técnicas de classificação. Conforme ilustrado na Figura 1, os atributos (ou características) que compõem o conjunto de entrada são constituídos pelas medidas extraídas dos logs de uso do sistema Moodle. Estas medidas foram obtidas a partir das interações e padrões de comportamento descritos na Seção 4.2. O rótulo para cada instância corresponde ao Estilo de Aprendizagem identificado por meio do questionário ILS aplicado aos estudantes no início do curso.

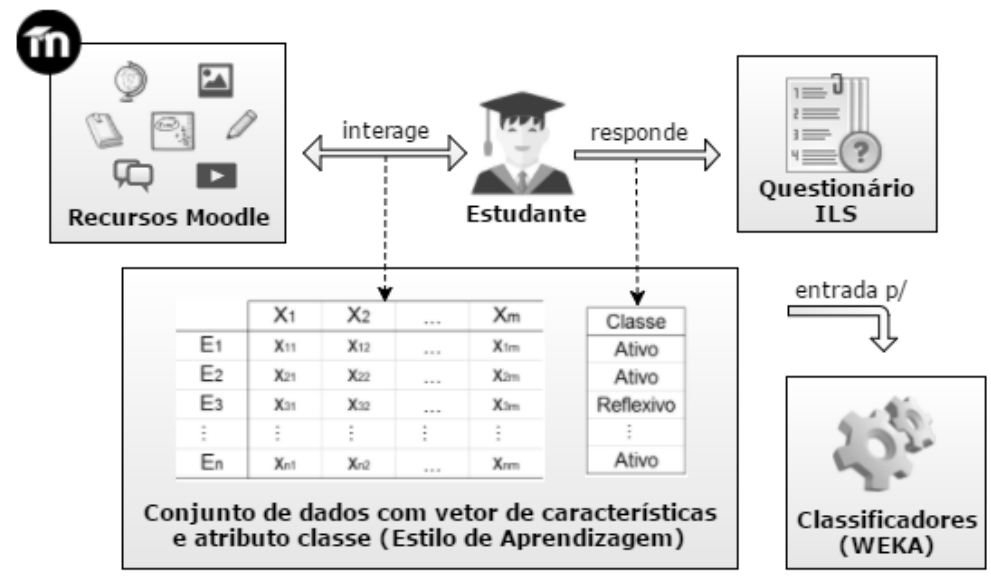

Figura 1. Metodologia para a classificação de estilos de aprendizagem.

\section{Experimentos}

Os classificadores foram avaliados para cada uma das quatro dimensões do modelo Felder-Silverman, ou seja, os mesmos conjuntos de atributos e valores serviram de entrada em quatro execuções distintas, alterando-se somente o atributo classe para cada execução. Sendo assim, a primeira execução foi configurada com o rótulo identificado para a dimensão de processamento (ativos $\mathrm{X}$ reflexivos), a segunda para a dimensão de captação (visuais $x$ verbais), a terceira para a dimensão de organização (sequenciais $x$ globais), e a quarta para a dimensão de percepção (sensoriais x intuitivos).

Diversos algoritmos foram avaliados, porém, dentre todos os experimentos realizados, neste trabalho serão apresentados somente os algoritmos que obtiveram os melhores resultados em cada paradigma do aprendizado de máquina. Para o paradigma estatístico foi selecionado o algoritmo Naive Bayes; para o paradigma baseado em instâncias foi selecionado o $I B k$; quanto ao paradigma conexionista foi selecionado o classificador MultiLayer Perceptron; e por fim, para o paradigma simbólico, foi escolhida a Árvore de Decisão J48.

\subsection{Pré-processamento de Dados}

Para cada execução, o conjunto de dados passou por um pré-processamento envolvendo dois filtros. Primeiramente, foi realizado um filtro de instâncias para balanceamento de classes por meio do método ClassBalancer. Neste filtro, são atribuídos pesos às instâncias do conjunto de dados para que cada classe tenha o mesmo peso total, mas de maneira que 
VI Congresso Brasileiro de Informática na Educação (CBIE 2017)

Anais do XXVIII Simpósio Brasileiro de Informática na Educação (SBIE 2017)

a soma total de pesos em todas as instâncias seja mantida. Esta prática serve como uma solução para problemas de classificação em conjuntos de dados com classes desbalanceadas. O segundo filtro foi o de seleção de atributos (AttributeSelection) que tem por objetivo selecionar um subconjunto de atributos a fim de reduzir a dimensão do conjunto de dados. Com essa redução de dimensão, reduz-se a quantidade de informação redundante e/ou espúria, aumentando a precisão dos classificadores; além disso, reduz-se a complexidade dos dados e assim o tempo de processamento para extrair deles algum conhecimento. Foram avaliadas as diversas técnicas, optando-se por usar o método WrapperSubsetEval, escolhido por demonstrar os melhores resultados quanto à precisão dos classificadores em relação às demais opções.

Em conclusão, todos os resultados demonstrados a seguir foram obtidos a partir da seguinte configuração: 1) filtro das instâncias com ClassBalancer; 2) seleção de atributos com WrapperSubsetEval; 3) execução do algoritmo em validação cruzada de 10 execuções (10-fold cross validation); e 4) computação das medidas Precisão, Revocação (Recall) e F-measure de cada uma das técnicas de aprendizado de máquina avaliadas.

\subsection{Resultados}

Como observado na Figura 2(a), o algoritmo Naive Bayes alcançou bons resultados para a predição automática de estilos de aprendizagem, especialmente quanto à dimensão de processamento. Neste caso, o método de aprendizado Bayesiano alcançou precisão de $79,4 \%$, revocação de 78,5\%, e F-Measure correspondente a 78,3. Estes resultados são viáveis para a aplicação do algoritmo em um cenário real de modelagem de estudantes.

Na Figura 2(b), são apresentados os resultados obtidos com o algoritmo MultiLayer Perceptron, pertencente ao paradigma conexionista no contexto de IA. Conforme pode-se observar, a técnica também alcançou bons resultados para a modelagem automática de estilos de aprendizagem, obtendo regularidade nos resultados para todas as dimensões. Com os percentuais de precisão variando entre 70,1\% (organização) e $75,1 \%$ (percepção), a técnica baseada em redes neurais mostra-se viável para aplicação, porém não superou as demais técnicas experimentadas.

Conforme ilustrado na Figura 2(c), o algoritmo IBk também alcançou bons resultados para a modelagem automática de estilos de aprendizagem, porém, diferentemente da técnica NaiveBayes, neste caso destacam-se os resultados quanto à dimensão de percepção. Foram alcançados os melhores resultados de classificação dentre todos os algoritmos, com 85,9\% de precisão, 84,6\% de revocação e F-Measure correspondente a 84,4 . Além disso, no geral, todas as classificações experimentadas com o IBk atingiram boas medidas, tornando o método viável em uma aplicação real.

Por fim, os resultados com a Árvore de Decisões J48 são apresentados na Figura 2(d). Como pode-se observar, esta técnica obteve resultados inferiores às demais avaliadas neste trabalho. Neste caso, esta classificação não é viável em um ambiente real, especialmente na dimensão de organização, a qual apresentou resultados similares ao de um classificador aleatório. A única exceção neste experimento foi a classificação quanto à percepção (sensoriais/intuitivos), que obteve precisão de 78,8\%, revocação de $78,7 \%$ e F-Measure correspondente a 78,6\%.

\subsection{Discussão dos Resultados}

Os resultados demonstraram que o aprendizado Bayesiano tem o melhor desempenho nas dimensões de processamento e de organização, ao passo que o aprendizado baseado 
VI Congresso Brasileiro de Informática na Educação (CBIE 2017)

Anais do XXVIII Simpósio Brasileiro de Informática na Educação (SBIE 2017)

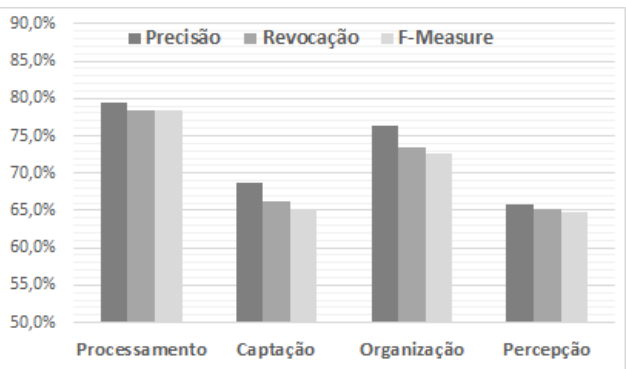

(a) Resultados Naive Bayes

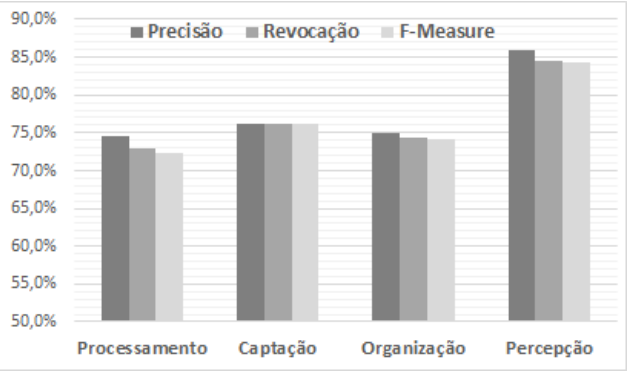

(c) Resultados Ibk

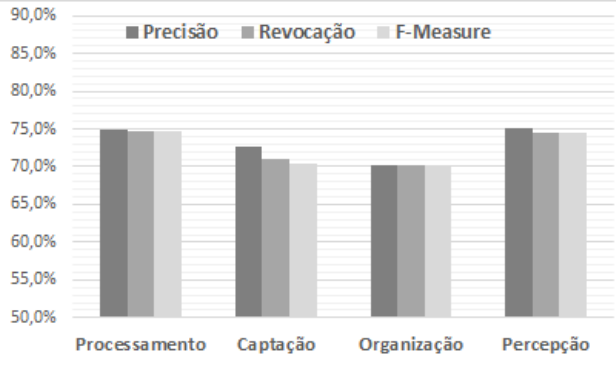

(b) Resultados MultiLayer Perceptron

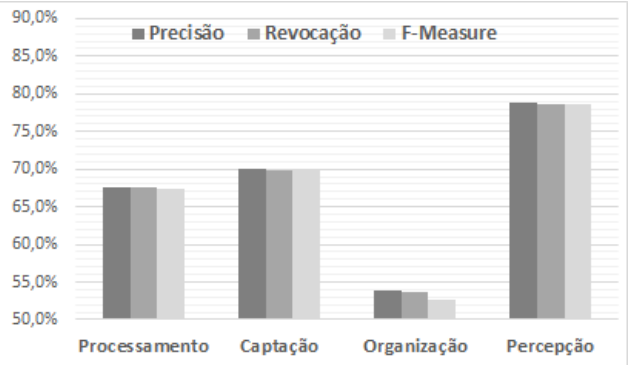

(d) Resultados J48

Figura 2. Resultados dos classificadores avaliados

em instâncias teve o melhor desempenho nas dimensões de captação e percepção. Em comparação com outros trabalhos, pôde-se notar que estes dois métodos, aprimorados com pré-processamento, superaram alguns dos trabalhos mais recentes, e mostraram-se viáveis para aplicação em um sistema educacional adaptativo. Portanto, o uso de um único classificador não é suficiente para todas as dimensões, de modo que o uso combinado dos aprendizados Bayesiano e baseado em instâncias provê uma classificação mais apurada.

No processo de classificação, a seleção de atributos e o rebalanceamento das classes impactaram significativamente o desempenho da predição, de maneira que estes dois pré-processamentos mostram-se necessários quando da identificação automática de padrões de aprendizado. Além disso, no que se refere às dimensões do modelo FelderSilverman, os resultados demonstraram que a dimensão de percepção foi a mais suscetível aos métodos de classificação, seguida das dimensões de processamento, organização, e captação. A avaliação e compreensão da previsibilidade de classificação de cada uma das dimensões é tema para futuros trabalhos.

\section{Conclusões}

Este trabalho trouxe esclarecimento com relação ao uso de técnicas de aprendizado de máquina na tarefa de caracterização de perfis de aprendizado. Especificamente, foram comparados quatro métodos de aprendizado de máquina voltados à tarefa de classificação: Naive Bayes, Multilayer Perceptron, Aprendizado baseado em instâncias, e Árvore de Decisão J48. Os métodos foram usados para predizer o perfil de aprendizado, segundo o modelo de Felder-Silverman, e O processo de classificação contou com uma etapa de balanceamento das classes seguida de uma etapa de seleção de atributos.

As conclusões apontam para o uso combinado de métodos de classificação de maneira a se obter melhor classificação em cada uma das dimensões de aprendizado, usando-se aprendizado Bayesiano e aprendizado baseado em instâncias. Estas técnicas 
VI Congresso Brasileiro de Informática na Educação (CBIE 2017)

Anais do XXVIII Simpósio Brasileiro de Informática na Educação (SBIE 2017)

obtiveram os melhores resultados, visto que o algoritmo Naive Bayes atingiu a melhor precisão para as dimensões de processamento $(79,4 \%)$ e organização $(76 \%)$ e o algoritmo IBk atingiu os melhores resultados para as dimensões de captação $(76 \%)$ e percepção $(85,9 \%)$. Além disso, as conclusões indicam a necessidade do uso de pré-processamento, evitando desbalanceamento de classes e atributos que não auxiliam na classificação; e para a compreensão sobre o quanto as dimensões de aprendizado podem ser classificadas automaticamente. Esta última conclusão remete a trabalhos futuros mais aprofundados.

De maneira geral, este trabalho contribui para o estado da arte com resultados de diversos classificadores aplicados ao mesmo problema de predição de EA, visto que esta trata-se de uma lacuna em trabalhos da área. Além disso, são expostos novos resultados que podem guiar publicações futuras, obtidos por meio de uma metodologia bem definida para extração de características em um ambiente virtual de ensino, pré-processamento de dados e coleta de diferentes medidas de avaliação dos resultados. A metodologia proposta também é entendida como uma contribuição ao estado da arte pelos autores, de modo que pode ser reproduzida em trabalhos futuros.

\section{Referências}

Coffield, F., Moseley, D., Hall, E., Ecclestone, K., et al. (2004). Learning styles and pedagogy in post-16 learning: A systematic and critical review.

Dorça, F., Lima, L., et al. (2011). Detecção e correção automática de ea em sistemas adaptativos para educação. Revista de Informática Teórica e Aplicada, 18(2):178-204.

Dung, P. Q. and Florea, A. M. (2012). An approach for detecting learning styles in learning management systems based on learners' behaviours. In International Conference on Education and Management Innovation, volume 30, pages 171-177.

Felder, R. M., Silverman, L. K., et al. (1988). Learning and teaching styles in engineering education. Engineering education, 78(7):674-681.

Feldman, J., Monteserin, A., and Amandi, A. (2015). Automatic detection of learning styles: state of the art. Artificial Intelligence Review, 44(2):157-186.

García, P., Amandi, A., et al. (2007). Evaluating bayesian networks' precision for detecting students' learning styles. Computers \& Education, 49(3):794-808.

Graf, S. (2007). Adaptivity in learning management systems focussing on learning styles. $\mathrm{PhD}$ thesis, Vienna University of Technology.

Graf, S., Liu, T.-C., et al. (2009). Supporting teachers in ident. students' ls in lms: An automatic student modelling approach. Journal of Educational Tech. \& Society, 12(4):3.

Keefe, J. (1979). Learning style: An overview. in national association of their relationship. British Journal of Educational Psychology, 67:199-212.

Kirschner, P. A. (2017). Stop propagating the learning styles myth. Computers \& Education, 106:166-171.

Sena, E. (2016). Proposta de uma abordagem computacional para det. autom. de estilos de aprendizagem utilizando modelos ocultos de markov e fslsm. Master's thesis, UFVJM.

Soloman, B. A. and Felder, R. M. (2005). Index of learning styles questionnaire. Available online at: http://www.engr.ncsu.edu/learningstyles/ilsweb.html, 70.

Truong, H. M. (2016). Integrating 1s and adaptive e-learning system: Current developments, problems and opportunities. Computers in Human Behavior, 55:1185-1193. 